\title{
Anomalous reduction of thermal conductivity in coherent nanocrystal architecture for silicon thermoelectric material
}

Yoshiaki Nakamura ${ }^{1,2}$, Masayuki Isogawa ${ }^{1}$, Tomohiro Ueda ${ }^{1}$, Shuto Yamasaka ${ }^{1}$, Hideki Matsui ${ }^{1}$, Jun Kikkawa $^{1}$, Satoaki Ikeuchi ${ }^{3}$, Takafumi Oyake ${ }^{4}$, Takuma Hori ${ }^{4}$, Junichiro Shiomi ${ }^{2,4}$, Akira Sakai ${ }^{1}$

${ }^{1}$ Graduate School of Engineering Science, Osaka University, 1-3 Machikaneyama, Toyonaka, Osaka 560-8531, Japan

${ }^{2}$ PRESTO, Japan Science and Technology Agency (JST), 4-1-8 Honcho, Kawaguchi, Saitama 332-0012, Japan

${ }^{3}$ ULVAC-RIKO Inc., 1-9-19 Hakusan, Midori-ku, Yokohama, Kanagawa 226-0006, Japan

${ }^{4}$ School of Engineering, The University of Tokyo, 7-3-1 Hongo, Bunkyo-ku, Tokoyo 113-8656, Japan

*Corresponding author: nakamura@ee.es.osaka-u.ac.jp (Y. Nakamura)

Reduction of thermal conductivity $\kappa$ while preserving high electrical conductivity $\sigma$ in materials continues to be a vital goal in thermoelectric study for the reuse of exhaust heat energy. In the use of an eco-friendly and ubiquitous element, $\mathrm{Si}$ as thermoelectric material, high $\kappa$ value in bulk $\mathrm{Si}$ is the essential bottleneck to achieve high dimensionless figure of merit. This is a motivation for many recent studies on reducing $\kappa$ in $\mathrm{Si}$, by nanostructuring, e.g., using grains/wires with size smaller than the phonon mean free path. However, $\kappa$ reduction that can be achieved tends to be saturated presumably due to an amorphous limit. Here, we present a nanoarchitecture for defeating the $\kappa$ amorphous limit while preserving bulk-like $\sigma$. This new nanoarchitecture is an assembly of Si nanocrystals with oriented crystals separated by a 1-monolayer amorphous layer with well-controlled nanoscale shaped interfaces. At these interfaces, novel phonon scattering occurs resulting in $\kappa$ reduction below the amorphous limit. Preservation of bulk-like $\sigma$ results from the coherency of the carrier wavefunctions among the oriented nanocrystals separated by the ultrathin amorphous layer. The results will bring environmentally-friendly and low-cost thermoelectric $\mathrm{Si}$ material compatible with mature LSI process technology and represent guidelines for optimized thermoelectric nanostructures.

Keywords: nano-structures, silicon, molecular beam epitaxy, thermal conductivity 


\section{Introduction}

In thermoelectric (TE) study for the reuse of exhaust heat energy and for cooling [1-6], materials with the high TE figure of merit has been explored. The dimensionless figure of merit, $Z T$ is written by $\left(S^{2} \sigma \kappa\right) T$, where $S$ is the Seebeck coefficient, $\sigma$ is the electrical conductivity, $\kappa$ is the thermal conductivity, and $T$ is the absolute temperature. The main approach to achieve high-ZT materials has been the use of heavy elements such as rare metal, e.g. $\mathrm{Bi}_{2} \mathrm{Te}_{3}$ and $\mathrm{PbTe}$ [7-9] despite the disadvantages of toxicity and high cost.

On the other hand, nanostructuring has recently become an important subject in TE study for control of thermal conductivity $\kappa$ independent of electrical conductivity $\sigma[2-6,10-11]$. In bulk chalcogenide materials such as $\mathrm{PbS}, \mathrm{PbTe}$, and $\mathrm{SrTe}$, nice works have been reported [12-14]. There have been many studies on developing high-ZT nanostructured-TE-materials composed of only $\mathrm{Si}$, an abundant and ecofriendly element for the realization of environmentally-friendly and low-cost TE materials [15-19]. Bulk Si shows low $Z T$ because of high $\kappa$ value despite its high power factor $\left(S^{2} \sigma\right)$. Therefore, lowering $\kappa$ by nanostructuring has been extensively attempted to realize high $Z T$. The efforts exhibited some success in the reduction of $\kappa$ from $\sim 150 \mathrm{Wm}^{-1} \mathrm{~K}^{-1}$ in bulk Si at room temperature (RT) to 48 (12) $\mathrm{Wm}^{-1} \mathrm{~K}^{-1}$ in non-doped $\left(10^{20} \mathrm{~cm}^{-3} \mathrm{~B}\right.$-doped) nanostructured bulk [18], $1.9 \mathrm{Wm}^{-1} \mathrm{~K}^{-1}$ in Si nanoscale mesh structures [19], and $\sim 1.5 \mathrm{Wm}^{-1} \mathrm{~K}^{-1}$ in Si nanowires [16]. However, this reduction becomes saturated at $\kappa$ values close to that of amorphous Si which is believed to be the lowest $\kappa$ (the amorphous limit). In sintered Si grains (nanostructured bulk $\mathrm{Si}$ ) or $\mathrm{Si}$ nanowires, the grain sizes/nanowire diameters were tuned to be smaller than the phonon mean free path, $\Lambda$ of bulk for the purpose of shortening the phonon scattering relaxation time by grain boundary scattering. Nevertheless, phonon grain/interface scattering at the nanoscale, essential for $\kappa$ reduction, has not been clarified. Conversely, there is room for finding novel phonon scattering at "a certain nanoscale-shaped interface" leading to drastic $\kappa$ reduction, which is unexpected in conventional theory. In terms of high $Z T$ in $\mathrm{Si}$, another important point is preserving high $\sigma$ of Si concomitantly with lowering $\kappa$. Note that it has been reported that the Seebeck coefficient, $S$ of Si was not changed so much by nanostructuring [18].

Here, we report a Si nanoarchitecture that has "well-controlled nanoscale-shaped interfaces" and oriented nanocrystals (NCs), which can defeat the amorphous limit of $\kappa$ while preserving bulk-like $\sigma$. As shown in Figure 1a, in the nanostructured Si, oriented Si NCs are separated by just a 1-monolayer (1-ML) 
thick amorphous layer, which leads to a coherent carrier wavefunction in addition to "well-controlled nanoscale-shaped interfaces." We find novel phonon scattering occurring at the interfaces depending on the interface curvature radius, while carrier wavefunction coherence results in preserving bulk-like $\sigma$. This study demonstrates that Si nanotechnology provides an innovative approach for high-TE-performance nanomaterials.

\section{Experimental Section}

\subsection{Sample growth}

The fabrication technique for connected Si NCs (CSN) with the same crystal orientation consisted of two basic processes: (1) $\mathrm{Si} \mathrm{NC}$ formation and (2) ultrathin $\mathrm{SiO}_{2}$ film formation as an amorphous barrier by slight oxidation of the NCs. These two processes were repeated to form CSNs, as shown in Figure 1a. The detail of fabrication is described below. Molecular beam epitaxy (MBE) was used for sample growth in an ultrahigh vacuum chamber equipped with an RHEED apparatus at a base pressure of $\sim 1 \times 10^{-8} \mathrm{~Pa}$. $\mathrm{Si}(001)$ substrates were introduced into the MBE chamber after chemical treatment and were degassed at $500^{\circ} \mathrm{C}$ for several hours. Clean $\mathrm{Si}(001)$ surfaces were then prepared by epitaxial growth of $\sim 100 \mathrm{~nm}$ Si buffer layers on the substrates at $500^{\circ} \mathrm{C}$ using an electron beam evaporator. Subsequently, the clean Si surfaces were oxidized at $\sim 500^{\circ} \mathrm{C}$ for $10 \mathrm{~min}$ at an oxygen pressure of $2 \times 10^{-4} \mathrm{~Pa}$ to form ultrathin $\mathrm{SiO}_{2}$ films with a thickness of $~ 1$ ML [20]; this process is referred to as "slight-oxidation" in this paper. The surface morphology was found to be unaffected by this process [21].

Next, x MLs of $\mathrm{Si}$ were deposited at $550^{\circ} \mathrm{C}$ on the ultrathin $\mathrm{SiO}_{2}$ films to form epitaxial $\mathrm{Si}$ NCs with an ultrahigh density $\left(>10^{12} \mathrm{~cm}^{-2}\right)$. At the first stage of Si deposition, nanowindows (NWs) less than $1 \mathrm{~nm}$ in size were created with an ultrahigh density in the ultrathin $\mathrm{SiO}_{2}$ films through the reaction $\mathrm{SiO}_{2}+\mathrm{Si} \rightarrow 2 \mathrm{SiO} \uparrow$, and these acted as nucleation sites for growth of spherical NCs. The Si NCs then grew epitaxially by coming in contact with the Si substrate through the NWs so that all of the NCs had the same crystal orientation as shown in Figure 1b [22] (See Chapter I in Supplementary material). A sharp NC size distribution [23] was obtained as shown in the inset in Figure 1b.The slight-oxidation process was then performed for the Si NCs. The pair of processes, $x$-ML Si NC formation and the slight-oxidization process, were repeated to form a stacked structure of the Si NCs ( $x$-ML-NC samples). We also confirmed almost no anisotropy of $\mathrm{NC}$ arrangement. 


\subsection{Characterization}

Thermal conductivity was measured by two different methods (see Chapters III-V in Supplementary material): (1) The $2 \omega$ method using an apparatus manufactured by ULVAC-RIKO Inc. (TCN-2 $\omega)$ [24], and (2) a time domain thermoreflectance (TDTR) method using femtosecond laser pulses [25]. The $2 \omega$ method is a means of measuring thermal conductivity along the cross-plane in a thin film based on the periodic heating method and the thermoreflectance method. The accuracy of this method is represented by the error values listed in Figure 3 and 4a (see Chapter IV in Supplementary material). Cross-sectional high resolution transmission electron microscopy (HRTEM) images were obtained along the [1 $\overline{1} 0]$ direction. RHEED observations were carried out using a $20 \mathrm{keV}$ electron beam with an incident direction of $\langle 110\rangle$.

For $\sigma$ measurements, the four terminal method was used in the lateral (surface) direction. Hall measurement was performed for $\mu_{\mathrm{H}}$ and carrier density. The Si NCs were randomly positioned; thus, we considered there to be no or negligible anisotropy of electrical conductivity in the CSN films. We confirmed the resistance of the non-doped Si substrate to be high enough that the electric current flowed only in the CSN film (see Chapter VIII in Supplementary material).

\section{Results and Discussion}

Figure 1c and its inset show a cross-sectional high-resolution transmission electron microscopy (HRTEM) image and reflection high energy electron diffraction (RHEED) pattern of the sample formed by 13 repetitions of 5-ML-Si NC formation. Figure 1c displays the macroscopic film morphology of the formed sample. In the microscopic view, the nanoscale dark region with the lattice image can be recognized, as shown in Figure 1d, which is an enlarged image of the rectangular region in Figure 1c. The outline shape of the dark regions with the lattice image seems to be round locally, as traced by the circles in Figure 1d. Fast Fourier transformation (FFT) patterns of the darker regions, as seen in Figure 1e, are the same as that of the Si substrate, indicating that these regions correspond to Si crystals with the same crystal orientation as that of the Si substrate. This is consistent with the RHEED pattern in the inset in Figure 1c indicative of epitaxial growth. $\mathrm{SiO}_{2}$ films were not observed at the interfaces between the $\mathrm{Si}$ NCs in the HRTEM images, presumably due to their thinness ( 1 ML). All of these results revealed that the CSN film contained randomly-stacked round-shaped NCs, almost all of which have the same crystal orientation, 
separated by ultrathin $\mathrm{SiO}_{2}$ film barriers as shown in Figure 1a. This well-controlled crystal orientation is completely different from the nanocomposite/nanostructured bulk composed of grains with random crystal orientation. It was possible to control the size of the round NCs by varying the deposition amount (10-20 ML) for NC sizes less than $10 \mathrm{~nm}$ (see Chapter I in Supplementary material). In the case of NCs larger than $10 \mathrm{~nm}$, the microstructure changed drastically due to NC coalescence.

Figure 2a shows a cross-sectional HRTEM image of a 46-ML-NC sample formed by 13 repetitions of $\mathrm{Si} \mathrm{NC}$ formation. The bright contrast bands with a width of $\sim 1 \mathrm{~nm}$ are considered to be amorphous $\mathrm{SiO}_{2}$ films, as indicated by the dotted lines in the Figure 2a. The HRTEM image in Figure 2a shows that the domed like regions that are $\mathrm{Si}$ crystals are separated by amorphous $\mathrm{SiO}_{2}$ layers. Figure $2 \mathrm{~b}$ shows an enlarged image near an amorphous $\mathrm{SiO}_{2}$ layer, which is indicated by the white arrow. In the inset, two FFT patterns are shown, corresponding to the dotted (A) and solid (B) square regions of the two different NCs in the HRTEM image. Both patterns are the same as that for the Si substrate, indicating that both NCs have the same crystal orientation as the substrate. Thus, as illustrated in the inset in Figure 2a, the domed Si NCs are about $40 \mathrm{~nm}$ and $13 \mathrm{~nm}$ in width and height, respectively, and they are stacked with the same crystal orientation despite the fact that they are separated by "amorphous" $\mathrm{SiO}_{2}$ layers.

We next investigated whether $\kappa$ could be controlled by a well-defined amorphous $\mathrm{SiO}_{2}$ layer $(1-\mathrm{ML}) / \mathrm{Si}$ interface with nanoscale-controlled curvature radius. Figure 3a shows the NC size (diameter or width) dependence of $\kappa$ for the present CSN film. Remarkably, $\kappa$ was found to depend on the NC size strongly, indicating the controllability of $\kappa$. It should be noted that for an NC size of $3 \mathrm{~nm}$, the $\kappa$ value was reduced to $0.78 \pm 0.12 \mathrm{Wm}^{-1} \mathrm{~K}^{-1}$ at $\mathrm{RT}$, which is lower than the values for other nanostructures [15-19] and is close to/below the amorphous limit of $\mathrm{Si}$ of $\sim 1-2.5 \mathrm{Wm}^{-1} \mathrm{~K}^{-1}$ [26-28] and that of amorphous $\mathrm{SiO}_{2}$ of $\sim 1 \mathrm{Wm}^{-1} \mathrm{~K}^{-1}$ [26]. The $\kappa$ value of amorphous Si has some variation. The recent paper reported that the $\kappa$ value depended on the formation temperature and thickness [29]. The $\kappa$ value under similar formation temperature and thickness of our CSN film is $0.98-0.99 \mathrm{Wm}^{-1} \mathrm{~K}^{-1}$ [29]. The consistency of the $\kappa$ values reported from several groups [26-29] exhibited the high reliability of its value. In order to investigate whether this anomalous reduction comes from unique properties of CSNs or from conventional interfacial thermal resistance and classical size (ballistic) effect [30] of Si thermal conductivity, we calculated $\kappa$ for a superlattice (SL) of amorphous $\mathrm{SiO}_{2}(\sim 0.3 \mathrm{~nm}) / \mathrm{Si}$ (thickness of $\left.t_{\mathrm{Si}}\right)$ under two different scenarios: fully diffusive (open triangles) and quasi-ballistic Boltzmann phonon transport (close triangles), by using the 
finite element and Monte Carlo methods [31], respectively as shown in Figure 3b. The ultrathin $\mathrm{SiO}_{2}$ films had $1 \mathrm{~nm}$ NWs as mentioned in "Experimental Section" as shown in the inset in Figure 3b, and the interfacial thermal resistance to $\mathrm{Si}$ was taken from the diffusive mismatch model (DMM) [32, 33]. The $\kappa$ values in the CSN films were found to be significantly smaller than those for the SL (triangle symbols). To discuss the $\kappa$ reduction effect per amorphous layer, we used HRTEM to count the number of $\mathrm{Si}_{\mathrm{SiO}} / \mathrm{Si}$ interfaces that heat flowed across in the vertical direction of the CSN film, $n_{\mathrm{i}}$. We then estimated the thermal resistance per amorphous layer $\left(\mathrm{Si} / \mathrm{SiO}_{2} / \mathrm{Si}\right)$ by dividing the thermal resistance of the CSN film by the average $n_{\mathrm{i}}$, as shown in Figure 4. The thermal resistance per amorphous layer of the SL, which was estimated from the calculation results in Figure 3b, was almost constant in this range, due to the constant interfacial thermal resistance based on DMM and strong phonon ballisticity. In contrast, the thermal resistance per amorphous layer for the CSN film was larger than that for the SL and depended on $t_{\mathrm{Si}}$, namely the curvature radius of the NCs. This unique increase in the thermal resistance per amorphous layer with decreasing $t_{\mathrm{Si}}$ caused an anomalous $\kappa$ reduction beyond the amorphous limit. This cannot be explained by conventional theory even if the ballistic phonon transport is considered by Monte Carlo simulations including first-principles-based interatomic force constants (Figure 4). The anomalous $\kappa$ reduction is presumably caused by a novel phonon scattering effect at the nanoscale-shaped interfaces determined by the amorphous layer covering the NCs, as shown in the inset in Figure 4. Therefore, the $\kappa$ reduction mechanism in the CSN film is completely different from that in conventional nanocomposites composed of smaller grains $(<\Lambda)[18,34]$ or a SL of Stranski Krastanov NCs [6], wherein the purpose of nanostructuring was to increase the interface/boundary number per unit volume. Using both the above conventional approach and our CSN results allows us to optimize the structure to reduce $\kappa$.

Because of the fact that almost all NCs in the CSN films have the same orientation and the amorphous interface layer is just 1-ML thick, the carrier wavefunction is expected to be coherent. We measured the electrical properties of the CSN film after doping by B-ion implantation (See Chapter VIII in Supplementary material). The hole mobility $\mu_{\mathrm{H}}$ in both the CSN film and bulk Si tends to decrease with the carrier density and saturate in the range larger than $\sim 10^{19} \mathrm{~cm}^{-3}$ [35], where the saturated $\mu_{\mathrm{H}}$ is $\sim 15(\sim 50)$ $\mathrm{cm}^{2} \mathrm{~V}-1 \mathrm{~s}^{-1}$ in CSN films (in bulk Si). The hole mobility in the CSN film saturates even at a low doping level of $\sim 10^{18} \mathrm{~cm}^{-3}$, presumably because of $\mathrm{NC}$ interfacial scattering, where the carrier scattering at interfaces is considered to be relatively suppressed due to the epitaxial growth. In bulk Si, reduction and saturation in $\mu_{\mathrm{H}}$ 
also occur because of the mean free path decreasing to the nanometer scale because of impurity scattering. Therefore, for high doping concentrations of $10^{19}-10^{20} \mathrm{~cm}^{-3}$, very little $\sigma$ degradation occurs in CSN films versus in bulk $\mathrm{Si}$.

The anomalously low $\kappa$ value below the amorphous limit achieved by precise control of the nanoscale interface is $\sim 200$ times smaller than that for bulk $\mathrm{Si}\left(\sim 150 \mathrm{Wm}^{-1} \mathrm{~K}^{-1}\right)$ at $\mathrm{RT}$, while $\mu_{\mathrm{H}}$ is reduced by 3 times. The CSN film is seen as an amorphous material for phonons and as a single crystal for carriers, which causes novel phonon scattering and a coherent carrier wavefunction as shown in Figure 1a. In general, the Seebeck coefficient, $S$, is insensitive to nanostructuring without quantum confinement [18]. Then, by adopting the $S$ value of p-type Si bulk $\left(\sim 220 \mu \mathrm{VK}^{-1}\right)$ [36] for a doping level of $\sim 1 \times 10^{20} \mathrm{~cm}^{-2}$, a $\sigma$ value of $\sim 190 \Omega^{-1} \mathrm{~cm}^{-1}$ (see Chapter VIII in Supplementary material), and the $\kappa$ value of $0.92 \mathrm{Wm}^{-1} \mathrm{~K}^{-1}$ (summation of lattice and carrier thermal conductivities), the dimensionless figure of merit $Z T$ was estimated to be about $\sim 0.3$ at RT, where the lattice thermal conductivity was adopted as $\kappa$ of non-doped sample ( 0.78 $\left.\mathrm{Wm}^{-1} \mathrm{~K}^{-1}\right)$ and carrier thermal conductivity was estimated as $\sigma L T\left(0.14 \mathrm{~W} \mathrm{~m}^{-1} \mathrm{~K}^{-1}\right)$ using Lorentz number $L$ (2.44×10-8 $\left.\mathrm{W} \Omega \mathrm{K}^{-2}\right)$. This is just a tentative estimation under poor conditions: non-optimized CSN doping, low temperature (RT), and ignoring the increase in $S$ due to quantum confinement in the NCs [36-40], which means we can expect higher ZT.

\section{Conclusion}

We have developed a CSN film with oriented crystals wherein ultrathin (1 ML) amorphous layers with controlled nanoscale curvature radius were introduced. Novel phonon scattering occurred at the nanoscale resulting in an anomalous $\kappa$ reduction below the amorphous limit. At the same time, $\sigma$ degradation was suppressed due to the coherent carrier wavefunctions. This will bring the environmentally-friendly and low-cost thermoelectric Si material and represent guidelines for an optimized thermoelectric nanostructure.

\section{Acknowledgements}

This work was supported in part by the JST PRESTO program. It was also supported by a Grant-in-Aid for Scientific Research B (25286026) and a Grant-in-Aid for Exploratory Research (25600016). This work was partially supported by TEPCO Memorial Foundation, Research Grant (Basic research). 


\section{Appendix A. Supplementary Material}

Supporting data associated with this article can be found in the online version at 


\section{References}

[1] G. J. Snyder, E. S. Toberer, Nat. Mater. 7 (2008) 105.

[2] R. Venkatasubramanian, E. Siivola, T. Colpitts, B. O’Quinn, Nature 413 (2001) 597.

[3] T. C. Harman, P. J. Taylor, M. P. Walsh, B. E. LaForge, Science 297 (2002) 2229.

[4] H. Ohta, S. Kim, Y. Mune, T. Mizoguchi, K. Nomura, S. Ohta, T. Nomura, Y. Nakanishi, Y. Ikuhara, M. Hirano, H. Hosono, K. Koumoto, Nat Mater. 6 (2007) 129.

[5] B. Poudel, Q. Hao, Y. Ma, Y. Lan, A. Minnich, B. Yu, X. Yan, D. Wang, A. Muto, D. Vashaee, X.Chen, J. Liu, M. S. Dresselhaus, G. Chen, Z. Ren, Science 320 (2008) 634.

[6] G. Pernot, M. Stoffel, , I. Savic, , F. Pezzoli, P. Chen, Nat Mater. 9 (2010) 491.

[7] D. A. Wright, Nature 11 (1958) 834.

[8] Z. H. Dughaish, Phys. B 322 (2002) 205.

[9] J. P. Heremans, V. Jovovic, E. S. Toberer, A. Saramat, K. Kurosaki, A. Charoenphakdee, S. Yamanaka G. J. Snyder, Science 321 (2008) 554.

[10] S. -M. Lee, D. G. Cahill R. Venkatasubramanian, Appl. Phys. Lett. 70 (1997) 2957.

[11] W. Kim, J. Zide, A. Gossard, D. Klenov, S. Stemmer, A. Shakouri, and A. Majumdar, Phys. Rev. Lett. 96 (2006) 045901.

[12] K. Biswas, J. He, Q. Zhang, G.Wang, C. Uher, Vinayak P. Dravid, and Mercouri G. Kanatzidis, Nature Chem. 3 (2011) 160.

[13] L. D. Zhao, S. -H. Lo, J. He, H. Li, K. Biswas, J. Androulakis, C.-I Wu,T. P. Hogan, D.-Y. Chung, V. P. Dravid, and M. G. Kanatzidis, J. Am. Chem. Soc. 133 (2011) 20476.

[14] K. Biswas, J. He, I. D. Blum, C.-I. Wu, T. P. Hogan, D. N. Seidman, V. P. Dravid, M. G. Kanatzidis, Nature 489 (2012) 414.

[15] D. Li, Y. Wu, P. Kim, L. Shi, P. Yang, A. Majumdar, Appl. Phys. Lett. 83 (2003) 2934.

[16] A. I. Hochbaum, R. Chen, R. D. Delgado, W. Liang, E. C. Garnett, M. Najarian, A. Majumdar, P. Yang, Nature, 451 (2008) 163.

[17] A. I. Boukai, Y. Bunimovich, J. Tahir-Kheli, J.-K. Yu, W. A. Goddard III, J. R. Heath, Nature $451(2008) 168$.

[18] S. K. Bux, , R. G. Blair, P. K. Gogna, H. Lee., G. Chen, M. S. Dresselhaus, R. B. Kaner, J.-P. Fleurial, Adv. Func. Mater 19 (2009) 2445. 
[19] J. -K. Yu, S. Mitrovic, D. Tham, J. Varghese, R. Heath, Nat Nanotech. 5 (2010) 718.

[20] A. A. Shklyaev, Y. Nakamura, M. Ichikawa, J. Appl. Phys. 101 (2007) 033532.

[21] T. Matsudo, T. Ohta, T. Yasuda, , M. Nishizawa, N. Miyata, S. Yamasaki, A. A. Shklyaev, M. Ichikawa, J. Appl. Phys. 91 (2002) 3637.

[22] A. A. Shklyaev, M. Ichikawa, Phys. Rev. B 65 (2002) 045307.

[23] N. Miyata, M. Ichikawa, Phys. Rev. B 70 (2004) 073306.

[24] R. Kato I. Hatta, Int. J. Thermophys. 29 (2008) 2062.

[25] D. G. Cahill, Review of Scientific Instruments, 75 (2004) 5119.

[26] D. G. Cahill, S. K. Watson, R.O. Pohl, Phys. Rev. B 46 (1992) 6131.

[27] D. G. Cahill, M. Katiyar, J. R. Abelson, Phys. Rev. B 50 (1944) 6077.

[28] Y. He, D. Donadio, G. Galli, Appl. Phys. Lett. 87 (2011) 144101.

[29] T. Zhan, Y. Xu, M. Goto, Y. Tanaka, R. Kato, M. Sasaki, and Y. Kagawa, App. Phys. Lett. 2014, 104, 071911.

[30] G. Chen, in Nanoscale Energy Transport and Conversion, Oxford Press, 2005.

[31] T. Hori, G. Chen, J. Shiomi, Appl. Phys. Lett. 104 (2014) 021915.

[32] E.T. Swartz, R.O. Phohl, Reviews of Modern Physics, 61 (1989) 605.

[33] H. Wang, Y. Xu, M. Shimono, Y. Tanaka and M. Yamazaki, Mat. Trans. 48 (2007) 2349.

[34] Y. Lan, A. J. Minnich, G. Chen, Z. Ren, Adv. Funct. Mater. 20 (2010) 357.

[35] D. K. Schroder, in Semiconductor Material and Device Characterization, Wiley, Hoboken, 3rd edn., 2006.

[36] O. Yamashita, N. Sadatomi, Jpn. J. Appl. Phys. 38 (1999) 6394.

[37] L. D. Hicks, M. S. Dresselhaus, Phys. Rev. B. 47 (1993) 16631.

[38] L. D. Hicks, M. S. Dresselhaus, Phys. Rev. B, 47 (1993) 12727.

[39] Y. Nakamura, K. Watanabe, Y. Fukuzawa, M. Ichikawa, Appl. Phys. Lett. 87 (2005) 133119.

[40] Y. Nakamura, A. Masada, M. Ichikawa, Appl. Phys. Lett. 91 (2007) 013109. 


\section{Figure captions}

$\begin{array}{ll}\text { Figure } 1 & \text { (a) Schematic of phonon scattering and coherent carrier wavefunction in CSN film. (b) }\end{array}$ plan-view STM image and RHEED pattern (right inset) of the plan view of 9 ML Si NCs epitaxially grown on a flat ultrathin $\mathrm{SiO}_{2}$ film on $\mathrm{Si}$ substrates. Histogram of $\mathrm{NC}$ size (left inset) indicating the diameter of $3.1 \pm 0.6 \mathrm{~nm}$. A sharp NC size distribution was confirmed and the NC density was ultrahigh $\left(\sim 2 \times 10^{12} \mathrm{~cm}^{-2}\right)$, corresponding to a NC-NC distance of $\sim 10 \mathrm{~nm}$. This density is 2 orders of magnitude higher than that of conventional Stranski-Krastanov NCs. (c) Cross-sectional HRTEM image and RHEED pattern (inset) of a 5-ML-NC sample formed by 13 repetitions of Si NC formation. (d) An enlarged image of the rectangle in (c). The average size of the NC was about $3 \mathrm{~nm}$ with a sharp distribution. (e) Fast Fourier transformation (FFT) images of an NC region in (d).

Figure 2 (a) Cross-sectional HRTEM image of the 42-ML-NC sample formed by 13 repetitions of $\mathrm{Si} \mathrm{NC}$ formation. The bright contrast bands indicated by dotted lines are considered to be $\mathrm{SiO}_{2}$ films; the contrast is due to the difference in the Si atomic density along the electron beam direction in the TEM observation. In the case of $\mathrm{NCs}$ surrounded by $\mathrm{SiO}_{2}$ films, the visibility of this contrast depended on the geometric curvature radius of the NCs. The width of the $\mathrm{SiO}_{2}$ films is seen to be about $1 \mathrm{~nm}$ in the HRTEM image due to the $\mathrm{NC}$ curvature; in reality the $\mathrm{SiO}_{2}$ film thickness is considered to be $<1 \mathrm{~nm}$. The inset is a schematic of dome-shaped stacked Si NCs. (b) Enlarged image near an amorphous $\mathrm{SiO}_{2}$ layer (white arrow) between two NCs. The inset shows two FFT patterns of the square regions in the lower left (A) and upper right (B).

$\begin{array}{ll}\text { Figure } 3 & \text { (a) Si crystal size dependence of thermal conductivity } \kappa \text { for the CSN (non-doped) film. }\end{array}$ The $\kappa$ values of the CSN film were measured by the $2 \omega$ method (closed circles) and time domain thermoreflectance (TDTR) method using a femtosecond pulse laser (open circles). Error bars of CSN with 3-5 $\mathrm{nm}$ size have almost the same size as those of the closed circles. The $\kappa$ values for other nanostructures are also shown (Nanostructured bulk Si: Ref. 18, Si nanomesh: Ref. 19, and Si nanowire: Ref. 16). (b) Comparison between the $\kappa$ values for the CSN film (experimental; circles) and a SL (calculated; triangles). The $\kappa$ values for the SL were calculated using the finite element (open triangles) and Monte Carlo (closed triangles) method, wherein the ultrathin $\mathrm{SiO}_{2}$ films had $\mathrm{Si}$ NWs of $1 \mathrm{~nm}$ size hexagonally arranged with a 
separation of $10 \mathrm{~nm}$, as shown in the inset in (b). This calculation includes interfacial thermal resistance in DMM. This SL with NWs is suitable for comparison with the CSN result because the present CSN had NWs in the ultrathin $\mathrm{SiO}_{2}$ films. The crystal size is the diameter of the round $\mathrm{Si}$ NCs or the width of the domed Si NCs in CSN film, and the Si layer thickness in the case of SLs. The errors display the accuracy of $\kappa$ measurement, which include the contribution of the uncertainties of physical values and measurement errors. (see Chapter IV in Supplementary material)

Figure 4 The dependence of thermal resistance per amorphous layer on the vertical length $\left(t_{\mathrm{Si}}\right)$ of the Si NCs in the CSN film (experimental; circles) or the Si layers in the SL (calculated; triangles). For round NCs smaller than $10 \mathrm{~nm}, t_{\mathrm{Si}}$ is equivalent to the diameters of NCs. The inset shows a schematic of conventional phonon scattering (SL) and novel phonon scattering (CSN) at the interfaces. 


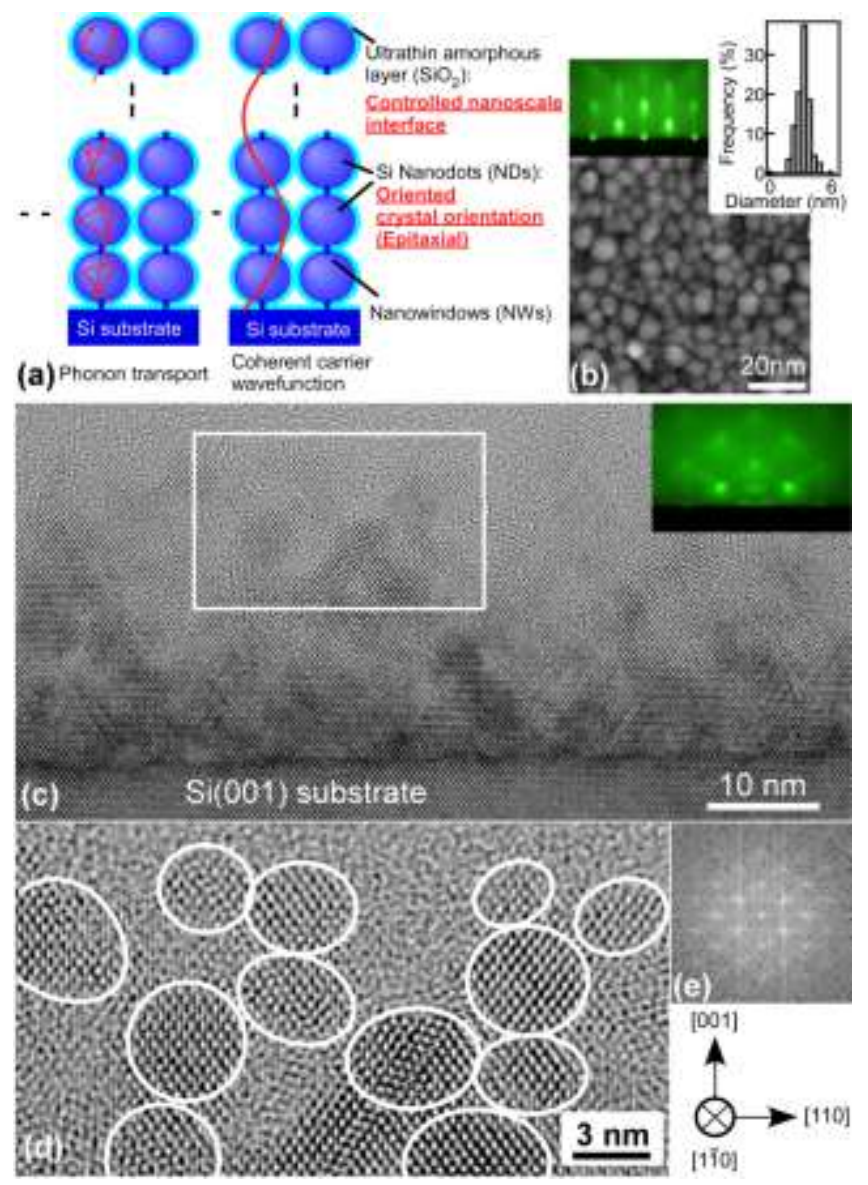

Figure 1. (a) Schematic of phonon scattering and coherent carrier wavefunction in CSN film. (b) plan-view STM image and RHEED pattern (right inset) of the plan view of 9 ML Si NCs epitaxially grown on a flat ultrathin $\mathrm{SiO}_{2}$ film on $\mathrm{Si}$ substrates. Histogram of NC size (left inset) indicating the diameter of $3.1 \pm 0.6 \mathrm{~nm}$. A sharp NC size distribution was confirmed and the NC density was ultrahigh $\left(\sim 2 \times 10^{12} \mathrm{~cm}^{-2}\right)$, corresponding to a NC-NC distance of $\sim 10 \mathrm{~nm}$. This density is 2 orders of magnitude higher than that of conventional Stranski-Krastanov NCs. (c) Cross-sectional HRTEM image and RHEED pattern (inset) of a 5-ML-NC sample formed by 13 repetitions of Si NC formation. (d) An enlarged image of the rectangle in (c). The average size of the NC was about $3 \mathrm{~nm}$ with a sharp distribution. (e) Fast Fourier transformation (FFT) images of an NC region in (d). 


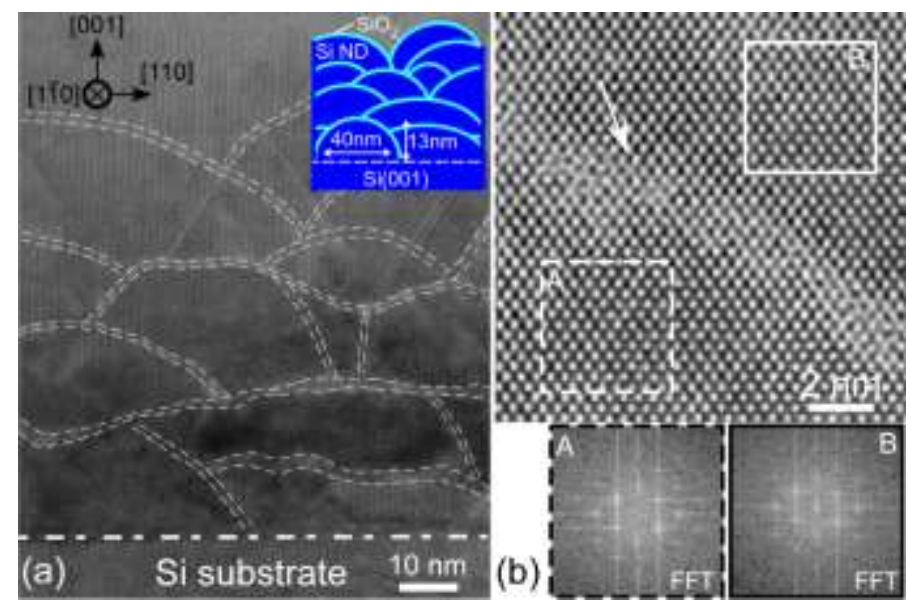

Figure 2. (a) Cross-sectional HRTEM image of the 42-ML-NC sample formed by 13 repetitions of Si NC formation. The bright contrast bands indicated by dotted lines are considered to be $\mathrm{SiO}_{2}$ films; the contrast is due to the difference in the Si atomic density along the electron beam direction in the TEM observation. In the case of $\mathrm{NCs}$ surrounded by $\mathrm{SiO}_{2}$ films, the visibility of this contrast depended on the geometric curvature radius of the NCs. The width of the $\mathrm{SiO}_{2}$ films is seen to be about $1 \mathrm{~nm}$ in the HRTEM image due to the NC curvature; in reality the $\mathrm{SiO}_{2}$ film thickness is considered to be $<1 \mathrm{~nm}$. The inset is a schematic of dome-shaped stacked Si NCs. (b) Enlarged image near an amorphous $\mathrm{SiO}_{2}$ layer (white arrow) between two NCs. The inset shows two FFT patterns of the square regions in the lower left (A) and upper right (B). 

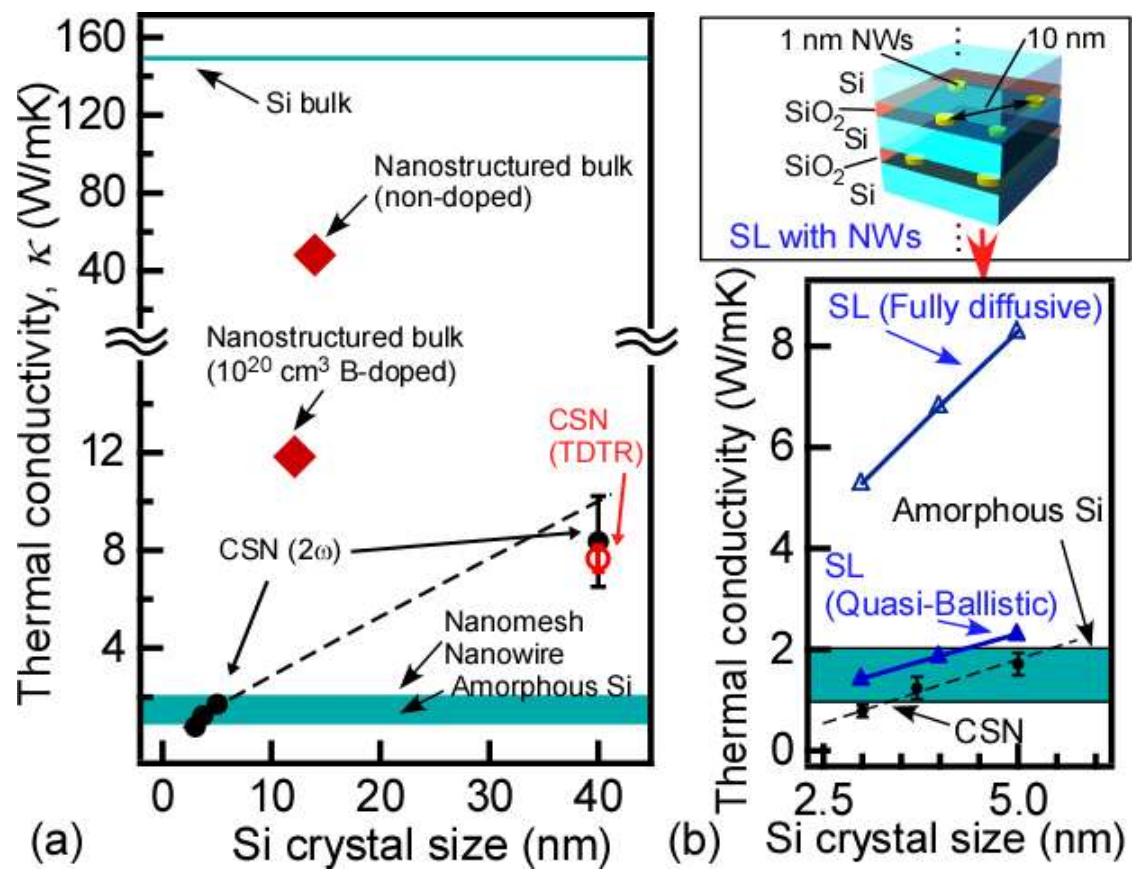

Figure 3. (a) Si crystal size dependence of thermal conductivity $\kappa$ for the CSN (non-doped) film. The $\kappa$ values of the CSN film were measured by the $2 \omega$ method (closed circles) and time domain thermoreflectance (TDTR) method using a femtosecond pulse laser (open circles). Error bars of CSN with 3-5 $\mathrm{nm}$ size have almost the same size as those of the closed circles. The $\kappa$ values for other nanostructures are also shown (Nanostructured bulk Si: Ref. 18, Si nanomesh: Ref. 19, and Si nanowire: Ref. 16). (b) Comparison between the $\kappa$ values for the CSN film (experimental; circles) and a SL (calculated; triangles). The $\kappa$ values for the SL were calculated using the finite element (open triangles) and Monte Carlo (closed triangles) method, wherein the ultrathin $\mathrm{SiO}_{2}$ films had $\mathrm{Si}$ NWs of $1 \mathrm{~nm}$ size hexagonally arranged with a separation of $10 \mathrm{~nm}$, as shown in the inset in (b). This calculation includes interfacial thermal resistance in DMM. This SL with NWs is suitable for comparison with the CSN result because the present CSN had NWs in the ultrathin $\mathrm{SiO}_{2}$ films. The crystal size is the diameter of the round $\mathrm{Si}$ NCs or the width of the domed Si NCs in CSN film, and the Si layer thickness in the case of SLs. The errors display the accuracy of $\kappa$ measurement, which include the contribution of the uncertainties of physical values and measurement errors. (see Chapter IV in Supplementary material) 


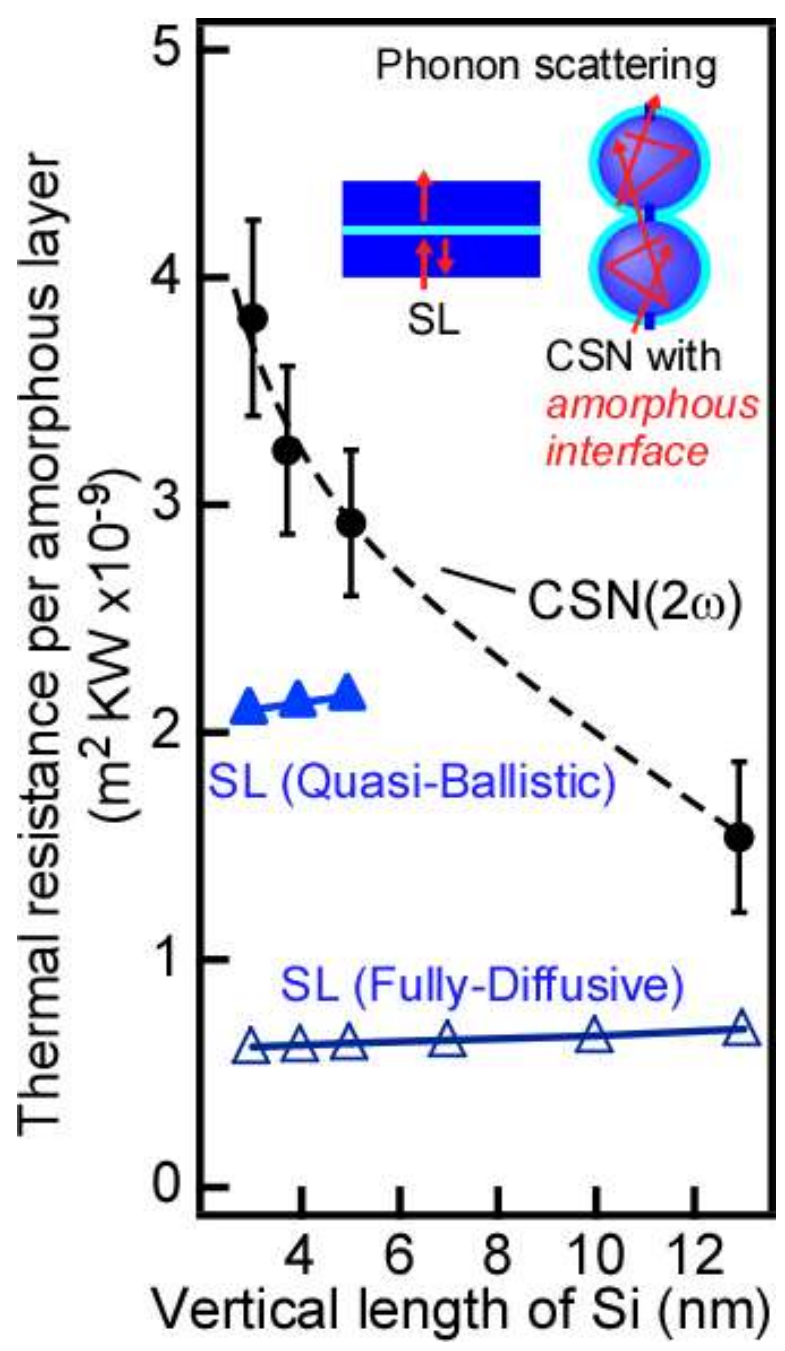

Figure 4. The dependence of thermal resistance per amorphous layer on the vertical length $\left(t_{\mathrm{Si}}\right)$ of the $\mathrm{Si}$ NCs in the CSN film (experimental; circles) or the Si layers in the SL (calculated; triangles). For round NCs smaller than $10 \mathrm{~nm}, t_{\mathrm{Si}}$ is equivalent to the diameters of NCs. The inset shows a schematic of conventional phonon scattering (SL) and novel phonon scattering (CSN) at the interfaces. 


\section{Personal portrait photo and biosketch}

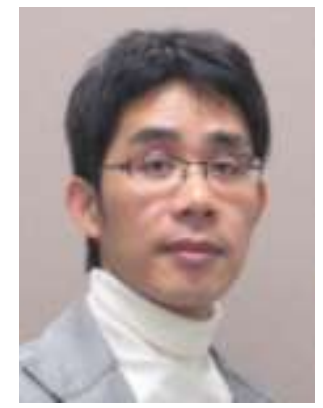

Yoshiaki Nakamura received his B. Eng. and M. Eng. from the University of Tokyo, Japan in 1997 and 1999, respectively, and obtained Ph. D. from Department of Applied Physics, Graduate School of Engineering, the University of Tokyo, Japan, 2002. He was also a PRESTO researcher, Japan Science and Technology Agency (2010-2014). His research covers fabrication and physical properties of nanostructures, surface science, molecular beam epitaxy. He is currently an associate professor at Osaka University, and works on development of Si-based thermoelectric nanomaterials.

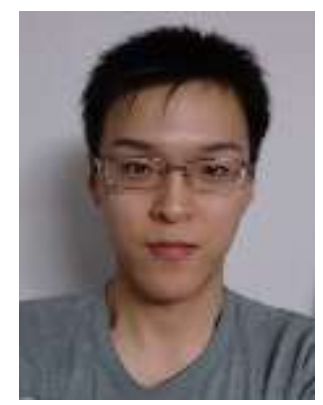

Masayuki Isogawa received his B. Eng. and M. Eng. from Osaka University, Japan in 2011 and 2013. His research is formation of novel nanostructure. He worked on the nanostructure growth using molecular beam epitaxy.

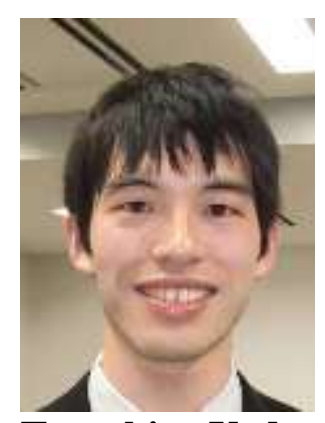

Tomohiro Ueda received his B. Eng. and M. Eng. from Osaka University, Japan in 2012 and 2014. His research covers the development of formation technique of stacked epitaxial Si nanodots and of its doping technique in the thermoelectric material field. 


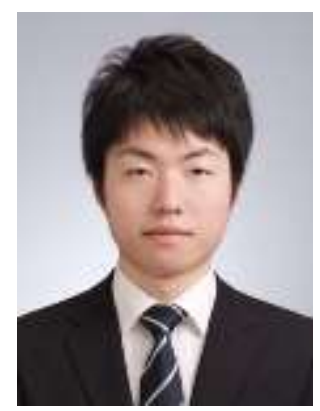

Shuto Yamasaka received his B. Eng. and M. Eng. from Osaka University, Japan, in 2011 and 2013 respectively. He is currently a Ph. D. Student in Graduate School of Engineering Science, Osaka University Japan. His current research interest focuses on fabricating Si based thermoelectric nanomaterials and on the thermal and electrical properties of them.

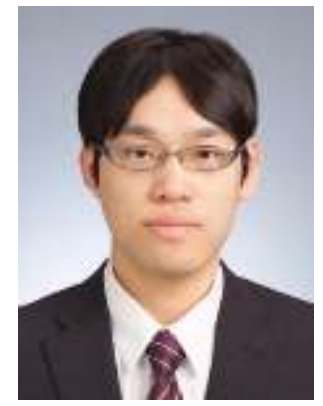

Hideki Matsui received his B. Eng. in School of Engineering Science from Osaka University, Japan in 2013. He is currently being in Graduate School of Engineering Science from Osaka University, Japan. His research interests focuses on electrical properties of nanostructured materials.

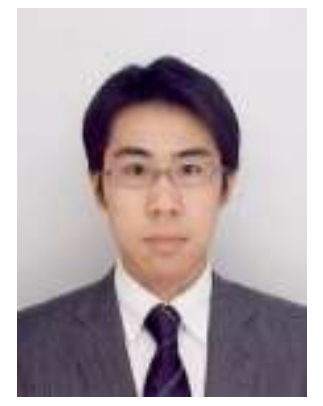

Jun Kikkawa received his B.S. (2001), M.S. (2003) and Ph. D. (2006) degrees from Osaka University. He was a postdoctoral fellow at National Institute of Advanced Industrial Science and Technology (2006-2008) and an assistant professor at Osaka University (2008-2012). He is currently a researcher from 2012 in National Institute for Materials Science and working on application of advanced electron microscopy. 


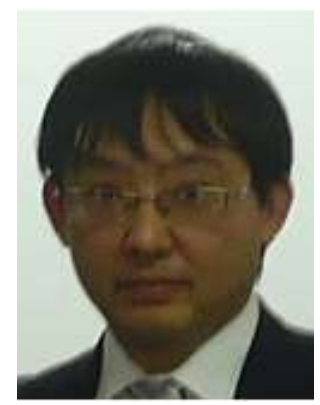

Satoaki Ikeuchi received his B. Sci. (2000) from School of Science, Osaka University, M. Sci. (2002), and Ph. D (2005) from Graduate School of Science, Osaka University. He researched in National Institute of Advanced Industrial Science and Technology (AIST) from 2005 to 2007. He belongs to ULVAC-RIKO Inc., from 2007. He is currently developing instruments of thermophysical property and calorimetry.

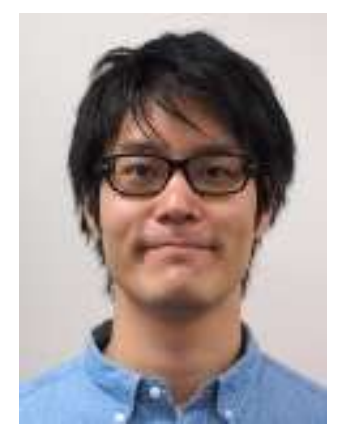

Takafumi Oyake received his B.Eng. (2012) and M. Eng. (2014) in Mechanical Engineering from The University of Tokyo, Japan. Currently, he is pursuing his Ph.D. there under Associate Professor Junichiro Shiomi. His research interests focus on experimental observations of thermal transport at nanoscale materials and interfaces, using optical experimental method.

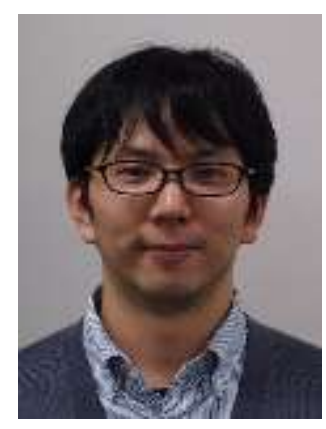

Takuma Hori received his B.S. (2010) and M.S.(2012) degrees in mechanical engineering from the University of Tokyo in Japan, and is currently a PhD. student there. His research interest focuses on studies of phonon transport in nanoscale thermoelectric materials using numerical simulations. 


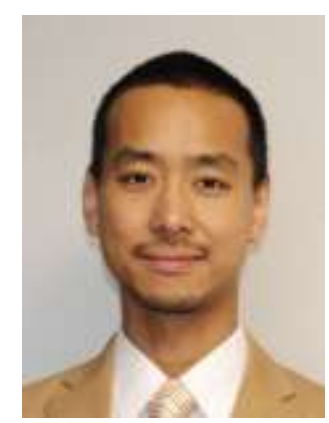

Junichiro Shiomi received the B.E. degree (1999) from Tohoku University, and Ph.D. degree (2004) from Royal Institute of Technology (KTH), Sweden. He is currently an Associate Professor in Department of Mechanical Engineering, The University of Tokyo. He is also a PRESTO researcher, Japan Science and Technology Agency. His research interests include heat conduction of nanomaterials, polymer composites, and thermoelectrics, phase change and fluidics in nanoscale, interfacial thermofluid dynamics, and thermal convections. He is a recipient of the Zeldovich Medal from the Committee on Space Research, and Young Scientists' Prize, the Commendation for Science and Technology by the Minister of Educational, Culture, Sports, Science and Technology.

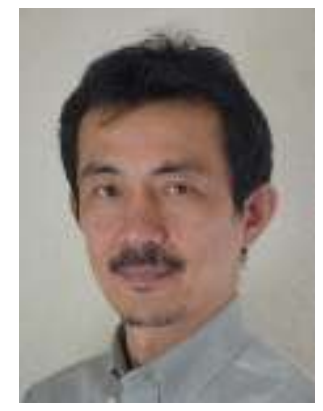

Akira Sakai obtained his B. Eng. (1984) and M. Eng. (1986) from Nagoya University, Japan. He received his Ph. D. from Department of Quantum Engineering in Nagoya University, Japan, in 1996. Currently he is a professor at Osaka University. His research interests include defect science and engineering of functional materials for electronic and optical devices and structural characterization of materials on a nanometer scale. 
A graphic for the Table of Contents

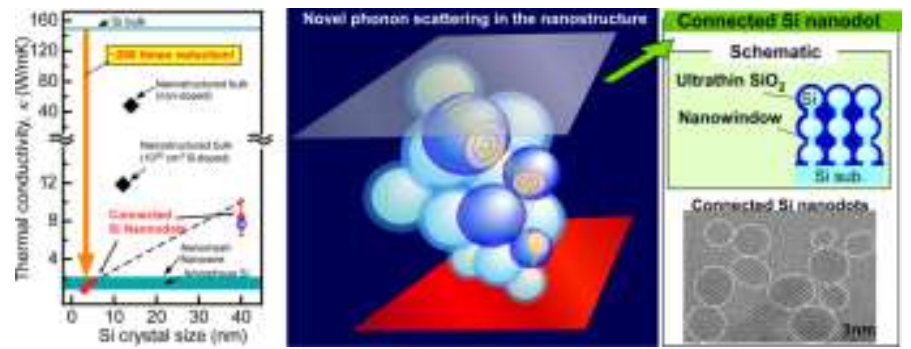

